\title{
Incidence of fracture in adjacent levels in patients treated with balloon kyphoplasty: a review of the literature
}

\author{
Peter G. Campbell · James S. Harrop
}

Published online: 7 December 2007

(C) Humana Press 2007

\begin{abstract}
The available evidence suggests that the treatment of painful vertebral compression fractures (VCFs) secondary to osteoporosis or multiple myeloma, by cement augmentation with balloon kyphoplasty (BK), is both safe and effective. However, there is uncertainty in the literature concerning the potential of the procedure to influence the risk for adjacent segment fracture. The aim of this article is to review the available peer-reviewed literature, regarding adjacent vertebral body fractures after kyphoplasty augmentation.
\end{abstract}

Keywords Kyphoplasty · Adjacent fracture - Vertebral body fracture $\cdot$ Vertebral compression fracture

\section{Introduction}

Vertebral compression fractures result from failure of the anterior column under loads either secondary to flexion or axial forces. They are not typically associated with neurological deficit, since the fractures do not extend into the spinal canal nor compress the neural elements. The majority of these fractures are associated with a limited period of pain. Most patients unknowingly sustain a fracture and thus never seek medical attention. In cases with persistent pain, medical management has been the mainstay of treatment for these fractures, which includes bed rest, analgesics, braces, and physical therapy. Generally, acute pain resolves in 4 weeks to 8 months, at times a spinal deformity may occur. These fractures might result from osteoporosis and

P. G. Campbell ( $\varangle)$ · J. S. Harrop

Department of Neurosurgery, Thomas Jefferson University

Hospital, 909 Walnut St, 3rd Floor, Philadelphia, PA 19107,

USA

e-mail: peter.campbell@mail.tju.edu relatively minor trauma or involvement of the vertebral body by an infiltrative tumor. Many of these fractures are not painful, but they seem to be associated with an impaired quality of life, an increase in mortality, and significant morbidity. Surgical treatment for symptomatic vertebral compression fractures had consisted in the past of reduction and internal fixation using an open anterior or posterior approach [1]. Results have often been compromised due to the extensive nature of the intervention and the poor bone quality secondary to osteoporosis, resulting in poor implant fixation and subsequent patient outcomes.

As minimal invasive spinal surgery techniques evolved, acute painful vertebral compression fractures were targeted for treatment through percutaneous procedures termed vertebroplasty or kyphoplasty. These procedures entail placing large spinal needles into the fractured vertebral body through a channel made in the pedicle and injecting bone cement into the fractured bone (Fig. 1). The term "kyphoplasty"' was introduced by Kyphon Inc. to describe balloon-assisted vertebroplasty. Unlike vertebroplasty, kyphoplasty aims not only to secure fracture fixation and stabilization, but also to reconstruct the vertebral anatomy and correct the kyphotic spinal deformity. A deflated balloon is inserted into the vertebral body through the pedicle and inflated to restore the height of a collapsed vertebral body and create a cavity inside. The balloon is then deflated and withdrawn. The remaining cavity is filled under low pressure with methylmethacrylate or bone substitute cement. This process is meant to stabilize the vertebrae, internally, which prevents the continuation of microfractures, and then facilitate pain relief while restoring the functional capacity. Restoring vertebral height and spinal alignment is believed to be important in the treatment of long-term increased morbidity and mortality that arises from vertebral compression fractures and spinal deformity. 
Fig. 1 Steps of kyphoplasty

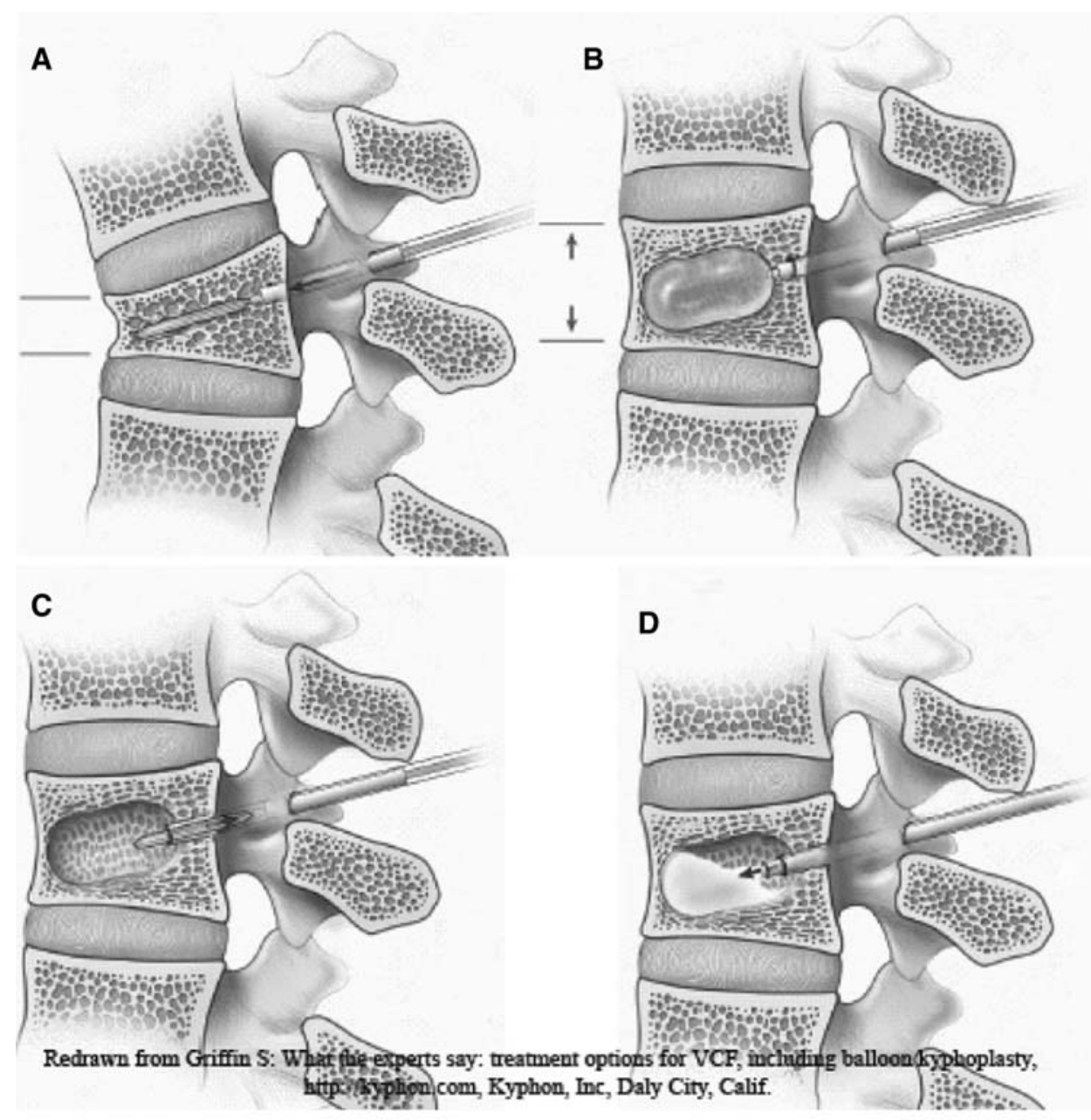

Early clinical results have illustrated significant pain relief and reduction of morbidity, but additional prospective studies are needed to refine the indications for the procedure. Potential complications of the procedure include: extrusion of the cement the spinal canal and subsequent spinal cord injury, infection, hematoma formation, pulmonary embolus, failure to relieve pain, and fracture of adjacent vertebral bodies. A kyphotic deformity increases the anterior stresses in adjacent levels by changing biomechanical loads as they are transferred through the spine. Therefore, a vertebral fracture is a potential increased risk for subsequent fractures in adjacent vertebrae [2]. For this reason, balloon kyphoplasty was introduced as a potentially more effective treatment option than vertebroplasty as it enabled the ability to restore the anterior part of the vertebral body and the biomechanical balance of the spine. As such, it has been presumed that treatment of compression fractures with kyphoplasty may reduce the rate of adjacent fracture. This article will attempt to review the current literature available regarding adjacent level fracture risk after kyphoplasty.

\section{Methods}

A review of the literature provided few studies to evaluate vertebral compression fractures in adjacent levels with kyphoplasty. A number of bibliographic databases were searched, including MEDLINE (Ovid), MEDLINE (R) Inprocess citations, and the Cochrane Library utilizing the keywords: vertebral body, adjacent fracture, vertebroplasty and kyphoplasty. Only one article specifically addressed the issue of adjacent level fracture after kyphoplasty. Several other articles reported adjacent fractures as a portion of their report on recurrent fracture after the procedures.

\section{Results}

Several studies were identified which focused on and directed conclusions about the association of adjacent fractures after a kyphoplasty procedure. In particular, Pflugmacher et al. [3] reviewed 42 patients with 67 
vertebral body fractures and focused their article on this issue. In their 2-year follow-up after kyphoplasty, an adjacent fracture occurred in $8(21.6 \%)$ patients with a total of $11(18.3 \%)$ new adjacent vertebral body fractures. However, one limitation of this study was it did not divide the patient's fractures into groups based on the primary mechanism of fracture.

Several other retrospective reviews extrapolate the risk of adjacent disease in their manuscripts on the complications after kyphoplasty. Uppin et al. [4] reviewed 177 osteoporotic patients treated with vertebroplasty specifically analyzing with subsequent fractures. With this method, 22 (12.4\%) patients developed 36 new fractures, and $24(66 \%)$ fractures occurred adjacent to the treated body. Fribourg et al. [5] treated 38 patients at 47 levels with balloon kyphoplasty. In the follow-up of 8 months, 10 patients had 17 subsequent fractures: nine at above-adjacent levels, four at below-adjacent levels, and four at remote levels. Harrop et al. [6] treated 225 vertebrae in 115 patients with balloon kyphoplasty. A total of 26 patients developed 34 subsequent fractures at an average follow-up of 1 month. In this study, the incidence of subsequent fractures was $11.25 \%$ in patients with primary osteoporosis, while it was $48.6 \%$ in patients with secondary osteoporosis due to steroid therapy. This review covered 80 patients with primary osteoporosis and 35 patients with secondary steroid induced osteoporosis. Hulme et al. [7] reported of the 12 reviewed studies on BK that presented data on fractures 766 patients had 115 new fractures and of those $66 \%$ were located at an adjacent level.

\section{Discussion}

Patients with severe intractable pain caused by compression fractures have few pain control options after medical management fails. Garfin et al. [8] enrolled a total of 155 elderly patients with symptomatic VCFs a prospective, multicenter treatment study of balloon kyphoplasty at 19 geographically diverse US centers. They reported a rapid, sustained pain reduction and functional improvement. Mean pain ratings decreased from 15/20 (visual analog scale) before surgery to $6 / 20$ within 7 days after kyphoplasty $(P<0.001)$. Hulme et al. [7] recently reviewed 69 clinical series evaluating both kyphoplasty and vertebroplasty and reviewed several aspects of their clinical outcomes. About 49-90\% reported an improvement in ambulation after treatment [7]. Out of the 12 reviewed studies on BK that presented data on fractures 766 patients had 115 new fractures and $66 \%$ were located at an adjacent level [7].

Kyphoplasty was developed after the use of vertebroplasty in an attempt to restore vertebral body height in addition to strengthening the body from its previous state. Height restoration has the potential benefit of reducing postfracture kyphosis, decreased pulmonary related mortality and possibly decreasing the incidence of adjacent level fractures [9]. In early biomechanical studies, both kyphoplasty and vertebroplasty restored body strength; however, kyphoplasty produced a statistically significant improvement in post-treatment vertebral body height [9]. In a systematic review of the two treatments, kyphopolasty produced a significant increase $(P=0.01)$ in vertebral height, with a mean value of $4.5 \pm 3.6 \mathrm{~mm}$. One can infer a $42 \pm 21 \%$ restoration of the height lost [10]. Pradhan et al. [11] reported a greater improvement in vertebral body height and decreased drop off in angular correction at 2 and 3 levels above and below the treated segments, thus implying greater correction in sagittal alignment (Fig. 2). A statistically significant decrease in adjacent level fracture has yet to be consistently reported in clinical studies.

There are several explanations for adjacent fractures after augmentation of the vertebral body. Rigid cement fixation could theoretically induce degenerative changes in adjacent bone, and the augmented vertebra is likely much stiffer than the adjacent vertebra [3]. Baroud et al. [12] developed biomechanical models to examine cement augmentation on the loading in adjacent vertebrae. In depth
Fig. 2 Pre- and postkyphoplasty lateral thoracic Xrays demonstrating a correction in the saggital alignment postoperatively
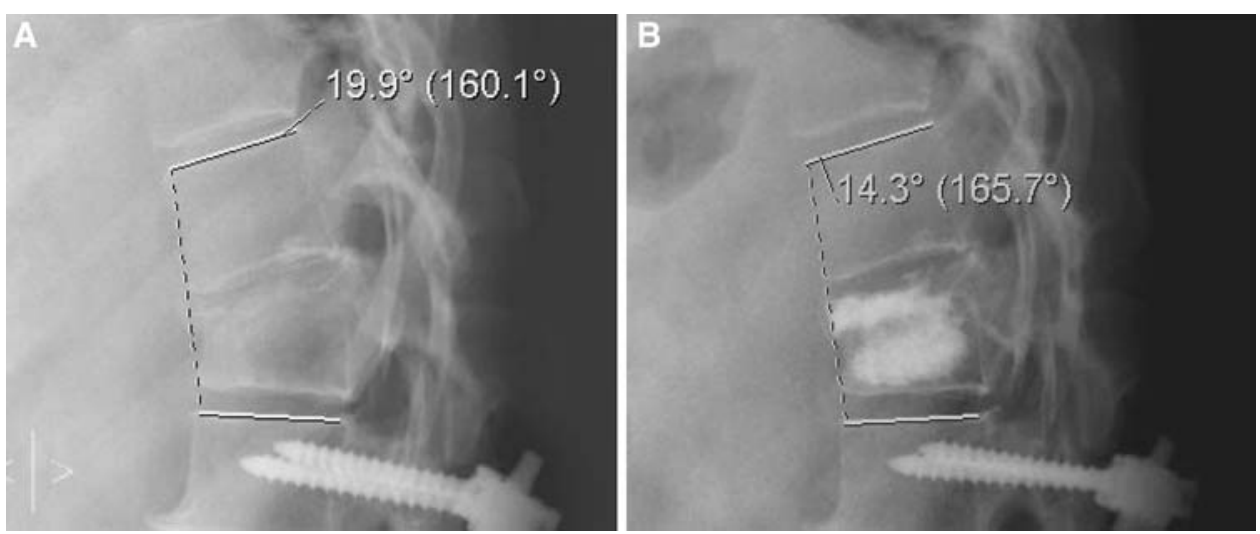
analysis of the model demonstrated that the cement in the treated vertebra acts as a pillar, reducing the physiologic inward bulge of the endplates. As a result of this effect, the pressure in the adjacent intervertebral disc increases by up to $19 \%$. The authors theorized that this shift in adjacent loading is one of the reasons for adjacent fractures. A statistically significant difference in adjacent fracture in kyphoplasty and vertebroplasty has yet to be consistently reported in clinical studies.

The amount of injected cement and also the restoration of vertebral height by kyphoplasty could have an influence the long-term outcomes of the procedure. However, these associations have not been demonstrated clinically [4].

In conclusion, balloon kyphoplasty is an effective, minimally invasive procedure for the stabilization of osteoporotic vertebral fractures leading to a statistically significant reduction of pain and functional status. Balloon kyphoplasty can stabilize and partially restore vertebral body height and is able to avoid further kyphotic deformities at the treated level. Starting antiosteoporosis therapy is essential and is the most effective measure in reducing the occurrence of further fractures [13]. There is insufficient evidence to conclude that kyphoplasty can reduce the incidence of adjacent fractures.

\section{References}

1. Terry Canale S. Campbell's Operative Orthopaedics. 10th ed. Chapter 35 CD-ROM. Mosby; 2002.

2. Yuan HA, Brown CW, Phillips FM. Osteoporotic vertebral deformity: a biomechanical rationale for the clinical consequences and treatment of vertebral body compression fractures. J Spinal Disord Techn 2004;17:236-42.
3. Pflugmacher R, Schroeder RJ, Klostermann CK. Incidence of adjacent vertebral fractures in patients treated with balloon kyphoplasty: two years' prospective follow-up. Acta Radiol 2006;47(8):830-40.

4. Uppin AA, Hirsch JA, Centenera LV, et al. Occurrence of new vertebral body fracture after percutaneous vertebroplasty in patients with osteoporosis. Radiology 2003;226:119-24.

5. Fribourg D, Tang C, Sra P, Delamarter R, Bae H. Incidence of subsequent vertebral fracture after kyphoplasty. Spine 2004;29:2270-6.

6. Harrop JS, Prpa B, Reinhardt MK, Lieberman I. Primary and secondary osteoporosis' incidence of subsequent vertebral compression fractures after kyphoplasty. Spine 2004;29:2120-5.

7. Hulme PA, Krebs J, Ferguson SJ, Berlemann U. Vertebroplasty and kyphoplasty: a systematic review of 69 clinical studies. Spine 2006;31(17):1983-2001.

8. Garfin SR, Buckley RA, Ledlie J. Balloon Kyphoplasty Outcomes Group. Balloon kyphoplasty for symptomatic vertebral body compression fractures results in rapid, significant, and sustained improvements in back pain, function, and quality of life for elderly patients. Spine 2006;31(19):2213-20.

9. Belkoff SM, Mathis JM, Fenton DC, Scribner RM, Reiley ME, Talmadge K. An ex vivo biomechanical evaluation of an inflatable bone tamp used in the treatment of compression fracture. Spine 2001;26(2):151-6.

10. Bouza C, Lopez T, Magro A, Navalpotro L, Amate JM. Efficacy and safety of balloon kyphoplasty in the treatment of vertebral compression fractures: a systematic review. Eur Spine J 2006;15(7):1050-67. Epub 2006 Jan 21. Review.

11. Pradhan BB, Bae HW, Kropf MA, Patel VV, Delamarter RB. Kyphoplasty reduction of osteoporotic vertebral compression fractures: correction of local kyphosis versus overall sagittal alignment. Spine 2006;31(4):435-41.

12. Baroud G, Nemes J, Heini P, Steffen T. Load shift of the intervertebral disc after a vertebroplasty: a finite-element study. Eur Spine J 2003;12:421-6.

13. Sorensen $\mathrm{OH}$, Crawford GM, Mulder H, Hosking DJ, Gennari C, Mellstrom D, et al. Long term efficacy of risedronate: a 5-year placebo-controlled clinical experience. Bone 2003;32:120-6. 